\title{
RECONSTRUCTION OF IP BEAM PARAMETERS AT THE ILC FROM BEAMSTRAHLUNG *
}

\author{
G. White, SLAC \& Queen Mary, University of London
}

\begin{abstract}
The luminosity performance of the ILC will be very sensitive to the parameters of the colliding bunches. Only some of these parameters can be measured using planned instrumentation. This analysis aims to access some of the colliding beam parameters not available by other means and to improve on the resolution of those that are. GUINEA-PIG is used to simulate the beam-beam interactions and produce beamstrahlung radiation $(\mathrm{e}+\mathrm{e}-$ pairs and photons). These are tracked to a simulation of the low-angle Beam Calorimeter and a photon detector and event shapes are produced. A Taylor map is produced to transform from the event shapes to the simulated beam parameters. This paper reports on the progress of this analysis, examining the usefulness of the proposed fitting technique.
\end{abstract}

\section{INTRODUCTION}

The requirements for high Luminosity at the ILC call for the focussing of the electron and positron bunches to tiny transverse dimensions (e.g. $553 \mathrm{~nm} \times 5 \mathrm{~nm}$ for the TESLA TDR parameters) [1]. The radiated energy as the colliding particles are accelerated in the huge EM fields present is called beamstrahlung. The total radiated energy is huge; about $108 \mathrm{TeV}$ per side, per bunch crossing. About $103 \mathrm{TeV}$ of the radiation is carried away by generated electron-positron pairs, the rest by the photons themselves. Based strongly on the preliminary analysis carried out by A. Stahl et. al. [2], this analysis attempts to use the relationship that exists between the properties of the colliding beams and the outgoing fluxes and distributions of the photon and pair radiation. By studying this relationship, it is hoped that a technique can be developed which allows one to reconstruct parameters of interest of the colliding beams.

\section{IP BEAM PARAMETERS}

There are a number of parameters that the colliding bunches have which affect the Luminosity. A total of 36 Interaction Point (IP) parameters are considered; the $\mathrm{x}, \mathrm{x}^{\prime}$, $y, y^{\prime}$ and $z$-sigmas, the energy and energy spread, the $x, x^{\prime}$, $y, y^{\prime}$ offsets, the $x$ and $y$ waist shifts, the bunch $x-y$ rotation angle, the number of particles and systematic $y-z$ distortions in the beams due to short-range wakefields in the accelerating structures and other accelerator elements, particularly the collimation sections. Most of the parameters are also split into mean and difference parameters between the electron and positron bunches.

\footnotetext{
* This work is supported by the Commission of the European Communities under the 6th Framework Programme "Structuring the European Research Area", contract number RIDS-011899.
}

\section{OBSERVABLES}

There are three sources of experimental observables considered here; the energy and number weighted distributions of the electron-positron pairs; the energy weighted photon distribution and the beam-beam kick (each beam experiences a strong transverse kick as it passes through the field of the colliding beam).

Using the ILC BeamCal $[1,3]$, situated just downstream of the final Quadrupole magnet $(3.65 \mathrm{~m}$ from the IP in the TESLA TDR design), event shape variables are formed based on the hits by electron-positron pairs. Event shape variables are also formed for the photons using a photon detector about $200 \mathrm{~m}$ downstream from the IP. The list of event shapes variables considered for the photon and pair detectors are as follows: Total Energy deposition; $r$ and $1 / \mathrm{r}$ moments; 'Thrust' axis and value (how 2-lobe like the radiation is); Angular spread; Ratio of radiation in inner and outer parts of the detector; left-right, top-bottom and diagonal asymmetries and ratio of Number of hits to total energy deposition for the BeamCal only. Together with the beam-beam deflection and splitting the variables into average and difference quantities between left and rightside detectors, this provides a total of 44 observables.

\section{BEAM \& DETECTOR SIMULATION}

In order to simulate the effect of varying beam parameters on the outgoing beamstrahlung, the code GUINEA-PIG [4] is used. The beam parameters are considered by generating an 80,000 macro-particle representation of the colliding bunches. In order to keep simulation times to a reasonable level, the electron and photon ratio parameters are set to $10 \%$. The routines for the remainder of this analysis are written in the Matlab environment, with the additional use of the Symbolic Math and Optimisation toolboxes.

The simulation of the BeamCal is based on the design specified in the TESLA TDR report [1]. It is modelled as a completely space filling area of similar sized crystals (1:1.2 width : height ratio) arranged in 7 concentric rings (total of 178 crystals). The front face of the detector is at a distance $3.65 \mathrm{~m}$ from the IP, the inner radius is at $1.2 \mathrm{~cm}$, the outer radius at $8 \mathrm{~cm}$. Pairs generated with GUINEAPIG are transported through a 4T solenoidal field and the number and energy of hits in the calorimeter crystal are stored. A full simulation of the calorimeter response, including energy smearing etc. is not provided here. Fringe solenoid fields are also not included here. The detector resolution is taken from the TDR estimates, $\Delta E[\mathrm{GeV}] \cong 10 \% \cdot \sqrt{E[\mathrm{GeV}]}$. Additional degradation occurs away from the central region of the detector which is ignored for this study. 
No existing design exists for the photon detector. Here I use a design based on some ideas set out in a note by Tauchi/Delarue [5]. I assume the detector is an ionization chamber detector with a thick copper end plate to convert photons to e-e+ pairs and contains a segmented pad readout. The detector is located $200 \mathrm{~m}$ downstream with the assumption that the main beam and charged pair background have been bent away. I assume the detector is a square geometry, centred on the nominal centre of mass of the photon flux and consists of 20x20 $1 \mathrm{~cm} 2$ readout pads. This is enough to constrain the photon flux through expected deviations from the nominal beam parameter set. Other (rather large) assumptions made at this stage include the perfect detection of photons above the pair production threshold in copper of about $30 \mathrm{GeV}$. Also, it is assumed here that the photon background that will be present at the lower end of the detection spectrum due to synchrotron radiation in the final focussing quadrupoles can be fully subtracted. It is also assumed that the detector can withstand the considerable power hitting it (several $\mathrm{kW})$. The resolution capabilities of this detector are assumed to be at the $10 \%$ level from reports of performance studies of similar detectors used elsewhere.

\section{BEAM PARAMETER RECONSTRUCTION TECHNIQUE}

The relationships between the observables and the beam parameters are both highly non-linear and nonorthogonal. This makes the reconstruction process difficult. In order to try to maximise the number of simultaneous parameters that can be measured, over as large an error range as possible, the following technique is suggested here: Construct a Taylor matrix series in terms of the deviation of the set of beam parameters under consideration from a pre-determined set of default values. A non-linear multi-parameter fitting algorithm should then be used to fit for the set of measured deviations of observables from their default set. The default parameter and observable set here is defined as the default TDR beam parameters and the resulting observables. This is defined mathematically below:

$$
\begin{aligned}
& x=\left[x_{1}, x_{2}, \ldots . x_{n}\right]^{\prime} \\
& x^{*}=x_{\text {Design }} \\
& f^{n}(x)=\text { observable } \mathrm{n} \text { for } \mathrm{x} \text { vector } \\
& f^{n}\left(x^{*}\right)=\text { observable } \mathrm{n} \text { for } \mathrm{x}^{*} \text { design vector } \\
& f^{n}(x)=f^{n}\left(x^{*}\right)+\left(x-x^{*}\right)^{\prime} \cdot \nabla\left[f^{n}\left(x^{*}\right)\right]+\frac{1}{2}\left(x-x^{*}\right)^{\prime} \widetilde{A}\left(x-x^{*}\right)+. \\
& \tilde{A}=\left[\begin{array}{cccc}
\frac{\partial^{2} f}{\partial x_{1}^{2}} & \frac{\partial^{2} f}{\partial x_{1} \partial x_{2}} & \cdot & \frac{\partial^{2} f}{\partial x_{1} \partial x_{n}} \\
\frac{\partial^{2} f}{\partial x_{2} \partial x_{1}} & \cdot & \cdot & \cdot \\
\frac{\partial^{2} f}{\partial x_{n} \partial x_{1}} & \cdot & \cdot & \cdot \\
\partial x_{n}^{2}
\end{array}\right]
\end{aligned}
$$

Where $\mathrm{x}$ is the vector of beam parameters, and $\mathrm{f}^{\mathrm{n}}$ is the nth observable, which is defined by the Taylor function in $\mathrm{x}$. The fit function then is described as the chi-squared statistic formed from the complete observable set:

$\chi^{2}=\sum_{o} \frac{\left(f^{o}(\underline{x})-O_{\text {measured }}\right)}{\sigma_{o}}$, where $\sigma_{\mathrm{o}}$ is the estimated error on the measured observable $\mathrm{O}$, really a function of $\mathrm{x}$.

\section{TESTING THE BEAM PARAMETER RECONSTRUCTION ALGORITHM}

A simulation environment was written to generate representations of the colliding bunches and run them through the beam-beam simulation and then generate the corresponding observables from the photon and pair files generated. All elements of the Taylor series were generated up to second-order, and diagonal-only terms where included up to ninth-order. All 44 observables were calculated for each GUINEA-PIG simulation run. To generate each Taylor series element, first to ninth-order gradients were calculated at the design point by running GUINEA-PIG to calculate the observables at 25 points around the design value. The gradients were symbolically calculated from polynomial fits to the data. The Hessian matrix was calculated from 2-D polynomial fits to all permutations of the 36 beam-parameters, mapped out on a $5 \times 5$ grid. In total, this required running GUINEA-PIG 16,575 times. On a $2.8 \mathrm{GHz}$ P4 CPU, an average of 240 seconds per GUINEA-PIG run and 200 seconds for the Matlab processing was experienced. Running on the Queen Mary High Throughput Computing Cluster [6], using 100 CPUs in parallel, this task took about 7 days. It is clear from this why we are limited to second-order calculations.

With the Taylor Matrix generated, tests were then performed to see if a multi-parameter fitter could be found to reconstruct a useful set of beam parameters.

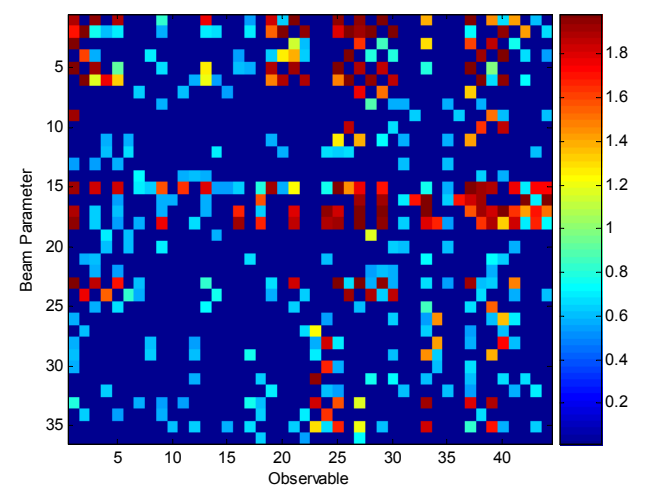

Figure 1: Correlation matrix between beam parameters and observables.

The correlation matrix between all beam parameters and observables is shown in figure 1 . The correlation coefficients are defined as $\mathrm{C}(\mathrm{i}, \mathrm{j}) / \mathrm{SQRT}\left(\mathrm{C}(\mathrm{i}, \mathrm{i})^{*} \mathrm{C}(\mathrm{j}, \mathrm{j})\right)$ where $C(i, j)$ is the covariance matrix for an observablebeam parameter pair. It can be seen how non-orthogonal the relationship between observables and beam parameters is. There are useful correlations between all parameters and observables to make use of however.

As a first test, only the first 10 beam parameters are considered. The other parameters are assumed to be nominal. These 10 are the $\mathrm{x}, \mathrm{y}, \mathrm{x}^{\prime}, \mathrm{y}^{\prime}$ and $\mathrm{z}$ sigmas (mean and differences). The fitting algorithm under test is the Matlab Optimisation Toolbox 'fmincon' routine [7]. This is a nonlinear constrained multi-parameter fitter which 
uses a 'Sequential Quadratic Programming' method (representing the state of the art in nonlinear programming methods- see references within [7]).

To test the fitting algorithm itself, 2 parameters are shifted from their nominal conditions and the algorithm is applied to see how close it fits the 2 parameters. No apriori knowledge is assumed as to which of the 10 parameters is in error or by how much. At this stage, the observables are assumed to be perfectly measured. The test is performed with all combinations of 2 errors from the 10 parameters, with low, medium and high error cases- corresponding to the parameters being 5, 25 and 40 $\%$ out from nominal.

The success of the fitting procedure was very sensitive to the set of constraints placed on the fit. An adequate fit can be found, however, by iterating through a set of constraints and choosing the resultant fit corresponding to the lowest fitted chi-squared (See figure 2).

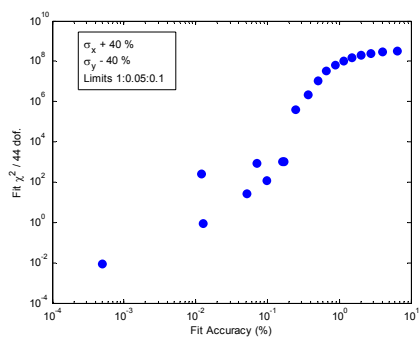

Figure 2: Fit accuracy vs. chi-squared for sigma-x and -y in error by $40 \%$ with varying constraints.

For all combinations of low and medium error cases, a fit accuracy of much better than $1 \%$ was always found. However, in about $20 \%$ of the high error cases the fit failed. The cases that failed were where the parameters in error were ones with high numbers of degenerate observable correlations. Here the fit gets lost in parameter phase space by trying to fit the wrong parameters and doesn't recover. The average time for the successful fits to converge (on a $3 \mathrm{GHz}$ P4) was 10 minutes.

In the cases where the fit fails, it is apparent from the poor chi-squared results that this has happened, so incorrect identification of beam parameters is not a problem. Better fit performances can be achieved by increasing the number of steps taken spanning the constraint space. Also, further constraints can be added by assuming some beam parameters will be measured at the ILC with wire scanners, bunch length monitors etc. It would also be possible to vary some parameters, looking at the fit for each one to further constrain the fitting. Obviously, this fitting technique will always be slow, even taking account of the expected increase in computing performance between now and when the ILC is commissioned. Hence, this can only be used as an offline analysis system. Although, assuming Moore's law holds up until the ILC turn-on date, one might expect fit times per bunch crossing (or average over many bunch crossings) of about 10 seconds.

As an example to asses the performance of the fit with realistic errors on the observables, see figure 3 . Here the horizontal and vertical beam spot sigmas were put in error (same small, medium and large cases as before). This time, the proper errors were added to all observables with 50 seeds for each parameter error case (normal distribution of errors assumed). Results could be improved by assuming an average over more bunches (better statistics), assuming the beam parameters remain reasonably stable over the averaged bunches.
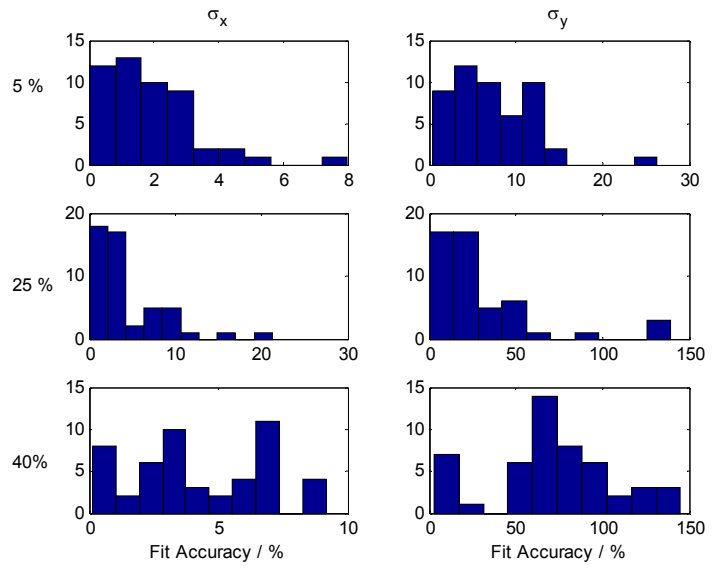

Figure 3: Accuracy of fit for $\mathrm{x}$ and $\mathrm{y}$ sigmas for 50 seeds, adding errors to all observables.

\section{FUTURE PLANS}

The next stage of this analysis is to look at the reconstruction performance when other beam parameters are away from nominal, including looking at some more realistic looking bunches that come out of ILC simulations (see my other submitted paper). It will also be seen how many of the 36 beam parameters it is reasonable to simultaneously fit and to try to develop a faster fitting technique. As an alternative technique, a neural network fitting procedure may also be tried.

\section{REFERENCES}

[1] TESLA TDR Chaps. 1 \& 4, TESLA Rep. 2001-23.

[2] A. Stahl "Luminosity Monitoring" $1^{\text {st }}$ ELAN Workshop INFN-LNF, Frascati, May 2004.

[3] W. Lohmann, "Calorimeters for Instrumentation of the Forward Region", LCWS2005, SLAC, California.

[4] D. Schulte, DESY-TESLA-97-08, 1997.

[5] N. Delarue, T. Tauchi, "Beamstrahlung monitoring of the beam beam effects at the Linear Collider", arXiv:physics/0408132, Aug 2004.

[6] http://194.36.10.1/cluster

[7] http://www.mathworks.com/access/helpdesk/help/ toolbox/optim/fmincon.html 